\title{
Molecular genetic tests in survival factors in patients with NSCLC in the clinical practice of Kazakhstan
}

\author{
Suriya Ye. Yessentayeva ${ }^{1}{ }^{*}$, Valeriy A. Makarov ${ }^{2}$, Zhanna A. Kalmatayeva ${ }^{2}$, Zhanar K. Zhakenova ${ }^{3}$, Dauranbek T. Arybzhanov ${ }^{4}$
}

Received: 12 Mar 2020

Published: 12 Oct 2021

Abstract

Background: Recent changes in understanding of the nature of cancer allow us, in some cases, to consider it a chronic process that requires constant or periodic treatment. The purpose of this study was to assess the efficacy of the methods for diagnosis and treatment of non-small cell lung cancer (NSCLC) in the Republic of Kazakhstan and present scientifically proven methods for the improvement of existing diagnostic algorithms and treatment programs.

Methods: This work was a retrospective study. A retrospective study using descriptive and analytical statistics was used as the main method. Reported data and medical records of the patients with NSCLC who were treated from 2015 to 2017 in 6 oncology clinics in the Republic of Kazakhstan were used as study materials. The methods used for histological studies and influence of the patient's sex on the frequency of various histological forms of NSCLC were studied. Polymerase chain reaction (PCR) studies to determine the epidermal growth factor receptor (EGFR) gene status as well as surgical methods were also studied.

Results: A comparative analysis of the compliance of oncologists from various regions of the republic with molecular genetic testing as an essential component of the diagnosis of NSCLC showed that the coverage of patients with immunohistochemical (IHC) and PCR studies in this country is low, $50.9 \%$ and $21.2 \%$, respectively. The study included data on 423 patients. At the same time, the majority of studies, $64.2 \%$ (IHC) and 100\% (PCR), were performed in patients in Almaty and only 35.8\% of IHC studies were performed in other 5 regions included in this study.

Conclusion: The morphological verification of malignant neoplasms in the lungs was based on histological studies. IHC and PCR coverage of the patients in the country was low. Most of the patients received pharmacotherapy. Surgical interventions were rarely performed. Also, the lack of IHC status data were a risk factor for the patients with NSCLC.

Keywords: Chronic Process, Histological Type, Malignant Process, Gene, Drug Therapy

Conflicts of Interest: None declared

Funding: The authors would like to acknowledge the contribution of the pharmaceutical company AO "Nobel AFF" for the allocated research grant under agreement No. 2 dated April 28, 2018 on rendering services for the implementa-tion of the project "Retrospective observational clinical study of the efficacy and safety of tyrosine kinase inhibi-tors (Erlotinib, Gefitinib) in the treatment of metastatic NSCLC in routine clinical practice in the Republic of Kazakhstan".

\section{*This work has been published under CC BY-NC-SA 1.0 license.}

Copyright $\odot$ Iran University of Medical Sciences

Cite this article as: Yessentayeva SYe, Makarov VA, Kalmatayeva ZhA, Zhakenova ZhK, Arybzhanov DT. Molecular genetic tests in survival factors in patients with NSCLC in the clinical practice of Kazakhstan. Med J Islam Repub Iran. 2021 (12 Oct);35:133. https://doi.org/10.47176/mjiri.35.133

\section{Introduction}

The problem of molecular genetic profiling in non-small cell lung cancer (NSCLC) in Kazakhstan has not been

\section{Corresponding author: Dr Suriya Ye. Yessentayeva, yessentayeva5560@tanu.pro}

1. Department of Oncology and Mammology, Kazakh-Russian Medical University, Almaty, Republic of Kazakhstan

2. Faculty of Medicine and Health Care, Al-Farabi Kazakh National University, Almaty, Republic of Kazakhstan

3. Department of Visual Diagnostics, S.D. Asfendiyarov Kazakh National Medical University, Almaty, Republic of Kazakhstan

4. Center for Chemotherapy and Centralized Chemotherapy, National Research Oncology Center, Nur-Sultan, Republic of Kazakhstan completely resolved. Underestimated significance of molecular genetic diagnosis in NSCLC in the regions results

个What is "already known" in this topic:

The majority of patients remain with an unidentified diagnosis, which is not detailed at the ultrastructural level, resulting in inadequate treatment and, as a consequence, low efficacy of pharmacotherapy and high mortality in this group of patients.

$\rightarrow$ What this article adds:

The lack of efficacy of morphological diagnosis and underestimation of the importance of molecular genetic diagnosis of NSCLC in the regions result in inadequate prescription of targeted therapy (TT) in patients with NSCLC, which affects low treatment efficacy and the inefficient use of budget funds. 
in inadequate choice of therapy in patients with NSCLC, which results in low treatment efficacy. The main purpose of this study was the evaluation of a real situation with the commitment of the physicians from the Oncological Service of the Republic of Kazakhstan to additional, but obligatory for the choice of treatment strategy, immunohistochemical (IHC) and polymerase chain reaction (PCR) studies.

Recent changes in understanding of the nature of cancer allowed us, in some cases, to consider it a chronic process that requires constant or periodic treatment. This became possible through a discovery of the basic mechanisms of its onset and development, which are a set of mutations. Therefore, the disease always begins with 1 "damaged" cell, which accumulated a number of disorders in it, and this underlies the understanding of the individual disease.

In Kazakhstan, long cancer (LC) has been a leader in the structure of cancer-related morbidity and mortality over the past 30 years. In 2012, it was the second after breast cancer. However, in terms of mortality, the rates remain the highest. LC-related mortality is $17.6 \%$ of all cancer-related deaths (1). Despite the fact that according to official data the proportion of patients with stage IV LC was $22.8 \%$ in 2015 , more than a half $(53.8 \%$ ) died because of an underlying disease over the first year after diagnosis, most of them at the stage of diagnosis. This indicates a higher rate of advanced forms of LC (1). NSCLC accounts for $85 \%$ to $90 \%$ of all cases of LC and the rates of SCLC in many countries have been steadily declining over the past 20 years (2). Moreover, an increase in the proportion of adenocarcinomas among all types of NSCLC has been observed. While back in the 90s, squamous cell carcinoma was a predominant histological type among all NSCLC forms, nowadays, $40 \%$ of patients are diagnosed with adenocarcinoma, $30 \%$ have squamous cell carcinoma, and $30 \%$ have large cell and other types of cancer (3-5).

Determining the histological subtype of LC, as well as the molecular genetic analysis for mutations, is an important criterion in the choice of therapy for LC. This principle is also reflected in the "LC Diagnosis and Treatment Protocols" approved in the Republic of Kazakhstan (6). It is known that targeted therapy (TT) is indicated, which should be prescribed as the first line therapy in patients with locally advanced or metastatic LC with a confirmed mutation of one of the genes, epidermal growth factor receptor (EGFR), anaplastic lymphoma kinase (ALK). However, to date, the problem of LC morphological verification (histological subtypes, molecular genetic testing for gene mutations) remains unresolved in Kazakhstan. According to 2015 data, the diagnosis of LC was verified only in $76.5 \%$ of cases, which indicates that diagnosis is established only based on clinical and radiological data in one-fourth of patients (1).

At the same time, an algorithm for diagnosing patients with a diagnosed "lung adenocarcinoma," which includes IHC, PCR, and fluorescence in-situ hybridization (FISH) studies to determine the EGFR and ALK gene status, is specified in the republican protocols (6). However, the analysis of the rates of examinations in the Republic of
Kazakhstan from 2014 to 2016 showed that in 2014, PCR study was performed in 31 (4.9\%) patients out of 626 cases with a histologically confirmed diagnosis of "adenocarcinoma," in 2015 in 109 (13.4\%) patients out of 815 cases of adenocarcinoma, and in 2016 in 181 (25.6\%) patients out of 708 cases (7).

A summary of diagnostic tests in patients to verify oncological diseases showed a general positive trend in the increase in cases of LC diagnosis; however, it is obvious that the majority of patients remain with an unidentified diagnosis, which is not detailed at the ultrastructural level, resulting in inadequate treatment and, as a consequence, low efficacy of pharmacotherapy and high mortality in this group of patients. On the other hand, an understanding of the importance and efficacy of TT in malignant tumors and the proven efficacy of tyrosine kinase inhibitors in NSCLC contributed to the fact that in 2012 these drugs were included in the Republican Protocols for the Treatment of LC and were included in the list of outpatient drugs purchased for for hospitals. However, the lack of efficacy of morphological diagnosis and underestimation of the importance of molecular genetic diagnosis of NSCLC in the regions result in inadequate prescription of TT in patients with NSCLC, which affects low treatment efficacy and the inefficient use of budget funds. The purpose of this study was to assess the efficacy of the methods for diagnosis and treatment of NSCLC in the Republic of Kazakhstan and present scientifically proven methods for the improvement of existing diagnostic algorithms and treatment programs. The objects of the study are diagnostic and treatment methods used in routine clinical practice in patients with NSCLC in the Republic of Kazakhstan.

\section{Methods}

This was a retrospective, observational, cohort study. A retrospective study using descriptive and analytical statistics was used as the main method. Reported data and medical records of the patients with NSCLC who were treated from 2015 to 2017 in the following oncological institutions were used as materials for the study:

1) Almaty Oncology Center, Almaty

2) East Kazakhstan Oncology Center, Semey;

3) Zhambyl Regional Oncology Center, Taraz;

4) Kyzylorda Regional Oncology Center, Kyzylorda

5) South Kazakhstan Regional Oncology Center, Shymkent

6) Aktobe Regional Oncology Center, Aktobe.

The subjects of the study were patients meeting the following criteria:

1. Women and men over 18 years with diagnoses of NSCLC.

2. Diagnosis can be confirmed morphologically (histology, cytology) and/or clinically and radiologically.

3. Patients diagnosed with NSCLC and receiving any type of antineoplastic therapy, including surgical intervention, radiotherapy, and pharmacotherapy as combination.

The study did not include data on patients with the following criteria: 
1. Children from 0 to 14 years old and adolescents under 18 years old.

2. Treatment naive patients with newly diagnosed NSCLC because of the presence of concomitant pathology or enrolled posthumously.

The following parameters were studied: age, gender, clinical stage (I, II, III, IV, unknown), histological type of cancer, IHC results, PCR results.

The following parameters were studied as objects of research: age, gender, clinical stage (I, II, III, IV, unknown), histological type of cancer, IHC results, PCR results.

Treatment data were also included in the study: surgical intervention, radiation, and pharmacotherapy (chemotherapy and targeted therapy). One of the important criteria of the analysis was the status at the end of the observation period:

- Date of the last patient information during the observation period.

- Survival status (alive, dead, unknown) at the end of the observation period.

- If the patient died before the end of the observation period: date of death; cause of death according to the attending physician (related to the tumor, other).

Patient data meeting the above criteria were recorded in the registry developed for this study. For the patients who met all inclusion criteria, all the necessary information was collected from their medical records, including demographic characteristics, oncological disease parameters, and information about the treatment, in compliance with the ethical principles of the Helsinki Declaration on $\mathrm{Pa}$ tient Safety (8) and the legislation of the Republic of Kazakhstan.

We used descriptive statistics for demographic and clinical characteristics of the patients with a confirmed diagnosis of NSCLC. The main diagnostic methods were evaluated. Information was collected on the main morphological methods to verify LC used in routine practice, with the evaluation of the frequency of molecular genetic tests and major reasons for their absence, data on the date of NSCLC diagnosis, beginning of treatment, data on the patient's condition at the end of the observation period, with an assessment of 1-, 2-, and 3-year survival, were collected.

The database was created in Microsoft Excel. Data statistical processing was performed with a personal computer using a statistical data processing program using the IBM SPSS Statistics 20 package (trial version). Survival rates were calculated from the beginning of treatment to all-cause death or to the date of the patient's last visit. The entire data set necessary for survival calculations was collected for 209 patients who were included in this study and received treatment at the Almaty Oncology Center (Almaty). Data collection was completed on January 1, 2019. The analysis of the survival of NSCLC patients was only possible for the Almaty Cancer Center (Almaty) data.

The main criterion for treatment efficacy in oncology, patient survival rate, was evaluated using the interval method, creating Life Tables, as recommended by the
Union for International Cancer Control (UICC) and the World Health Organization (1979). Overall survival using Life Tables and time to median survival in months were evaluated. In all cases, survival curves were obtained. The Kaplan-Meier method and the Forest plot were used. The significance was evaluated using the log-rank test.

Since the data obtained during the study were normally distributed, parametric tests were used for statistical analysis, a probability of $95 \%$ was determined to exclude the systematic error of the study, and the Pearson $\chi 2$ test was used to compare the differences. The impact of various factors on survival was assessed after calculating the relative risk (RR) of death.

A total of 423 questionnaires (including those for 209 patients of the Almaty Cancer Center) corresponding to the above criteria were selected for statistical processing, which were subject to random sampling.

\section{Results}

The study included data on 423 patients who received treatment in 6 oncology clinics in the Republic of Kazakhstan, including 309 (73\%) men and 114 (27\%) women. The mean age of male patients was \pm 57 years (range, 32 to 82 years). The mean age of female patients was \pm 60 years (range, 33 to 86 years). In $93.6 \%$ ( $\mathrm{n}=396$ patients) of the patients diagnosed with NSCLC, the diagnosis was done from 2015 to 2017 , in $4 \%$ ( $n=17$ patients) in 2014 , and single patients with this diagnosis were found from 2006 to 2013.

Data analysis for the patients with LC according to the rate of the primary process is presented for 421 patients; data on the stage of the disease is absent in $2(.5 \%)$ cases only. In the initial diagnosis of malignant neoplasms in the lungs, early stages were detected in $37.4 \%$; the majority of patients, $62.0 \%$, initially had a locally advanced or metastatic process (Table 1).

However, according to the Oncology Service data for 2016 (4), the proportion of patients with stage I to II LC was even lower, $26.5 \%$, that is, 2 of 3 patients had an advanced disease that could not be treated with radical methods. In this group of patients, pharmacotherapy and, in some cases, radiation therapy are indicated. A summary of data on clinical and demographic characteristics of the patients showed that men were diagnosed with LC 3 times more often. In most patients, the disease was diagnosed at an advanced stage, but despite the small number of patients with primary metastatic process (68 patients, $16.1 \%$ ), according to the general condition of the patients, almost half $(\mathrm{n}=222$ patients; $52.6 \%$ ) had an Eastern Co-

Table 1. Distribution of the patients with lung cancer according to distant metastases

\begin{tabular}{lcc}
\hline Stage of the Disease & Number of Patients & $\%$ \\
\hline I & 41 & 9.7 \\
II & 117 & 25.7 \\
III & 194 & 45.9 \\
IV & 68 & 16.1 \\
Total & 420 & 99.3 \\
No data & 3 & 0.7 \\
Total & 423 & 100 \\
\hline
\end{tabular}


operative Oncology Group (ECOG) scale score of 2 to 3, which might affect the choice of treatment.

\section{Morphological Diagnosis of Malignant Neoplasms in the Lungs}

Currently, the treatment strategy for patients diagnosed with LC is based primarily on the results of the morphological histotype of the tumor, followed by its molecular genetic profile in certain forms. This principle underlies individual therapy. According to our analysis of 423 cases, a cytological study was conducted in 123 (29.1\%) patients: in $13(10.6 \%)$ patients, tumor elements were not found in the cytological preparation. In $5(4.1 \%)$ patients, a malignant neoplasm was suspected, including 1 patient with suspected malignant mesothelioma. Also, 105 $(85.4 \%)$ patients were diagnosed with cancer, while squamous cell carcinoma was diagnosed in $34.9 \%$, lowdifferentiated tumor in $11.4 \%$, a malignant process without differentiation was cytologically confirmed in $14.6 \%$, and only $4.9 \%$ of patients had adenocarcinoma.

A histological examination is still the grounds for morphological verification of malignant neoplasms. Valid histological examination data were available in 413 $(97.6 \%)$ cases. Data were absent in $10(2.4 \%)$ patients. In fact, histological material for morphological examination was obtained in 390 (92.2\%) patients; in 33 (7.8\%) cases, this type of study was not performed. According to morphological studies, of 390 cases of suspected malignant process in the lungs, a histological diagnosis was confirmed in $385(98.7 \%)$ cases; histological data for the malignant process was not obtained in $5(2.3 \%)$ patients.

Of 385 patients, $2(0.5 \%)$ had small cell lung cancer (SCLC) and $4(1 \%)$ had neuroendocrine tumors (carcinoid); in 29 (7.5\%) cases, tumor histotype was not identified with the differentiation of a "malignant tumor" or a "malignant tumor metastasis". Undifferentiated cancer was diagnosed in $16(4.2 \%)$ patients, and sarcomotoid clear cell tumor in $4(1.0 \%)$ patients (Fig. 1). The most commonly diagnosed cancer was NSCLC, identified in $318(82.6 \%)$ cases. Among the morphological forms of NSCLC, squamous cell type $(n=196$ patients; 61.6\%) was most common; glandular squamous cell carcinoma was identified in $5(1.6 \%)$ patients, and large cell type of squamous cell cancer was identified in $2(0.6 \%)$ cases. Pulmonary adenocarcinoma was identified in 104 (32.7\%) patients and bronchoalveolar cancer in 11 (3.5\%) cases (Fig. 2).

At the next step, we studied the frequency of various

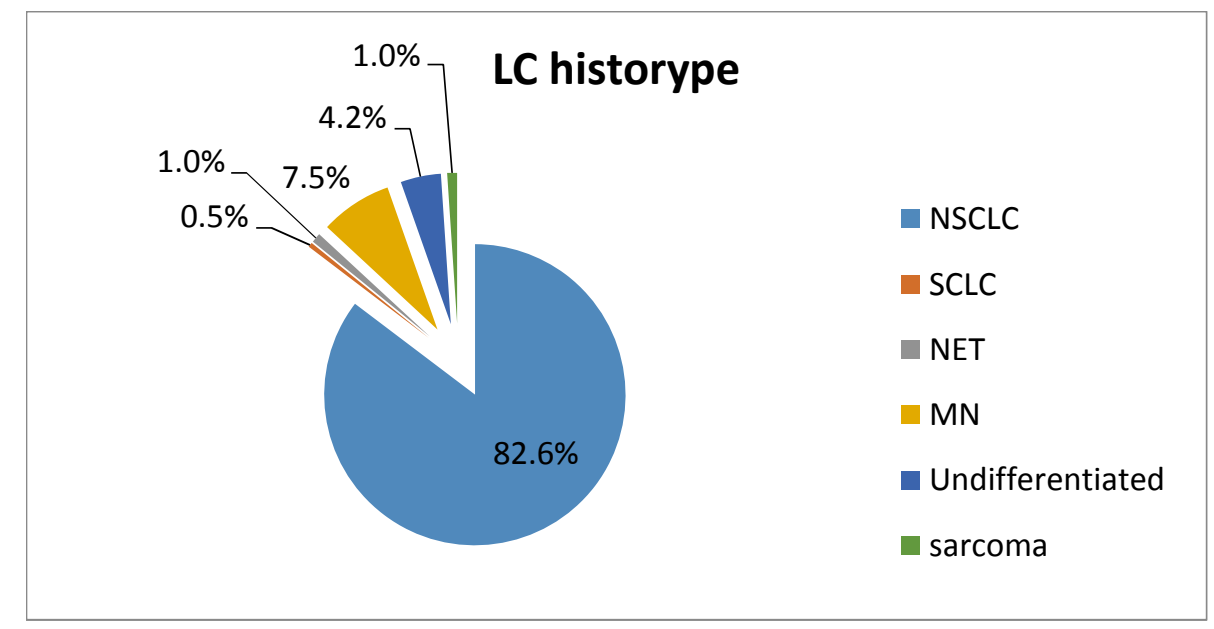

Fig. 1. Morphological histotypes of malignant lung tumors

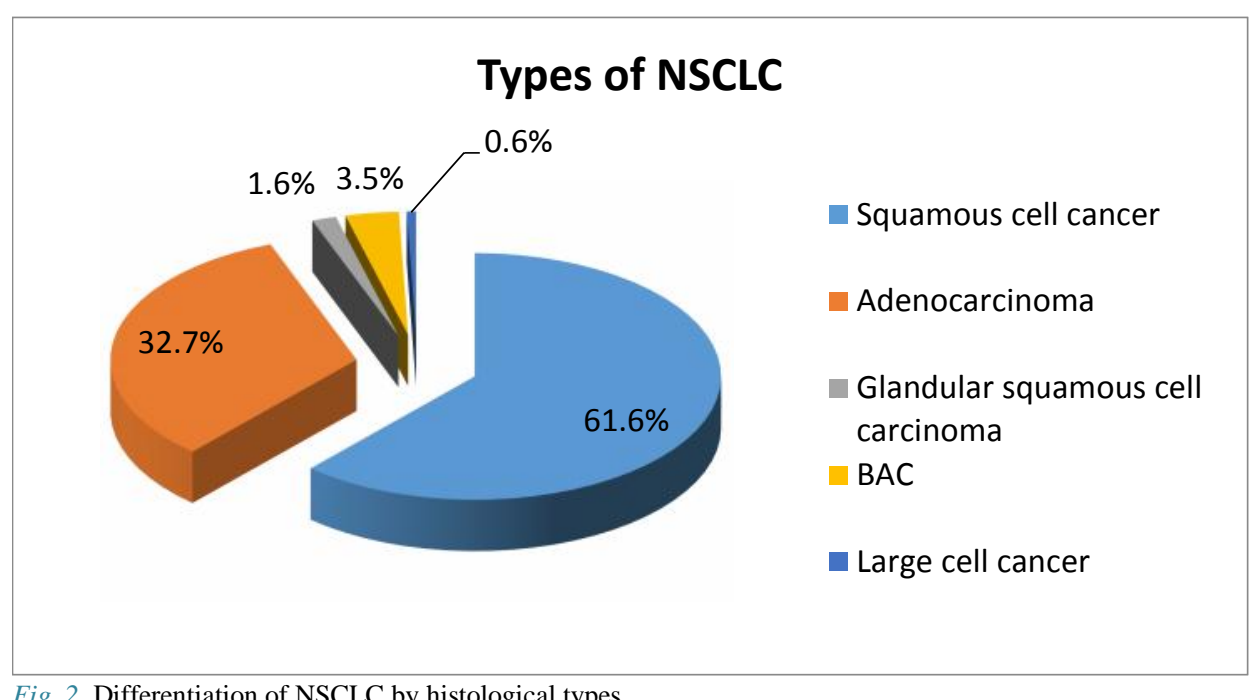

Fig. 2. Differentiation of NSCLC by histological types 


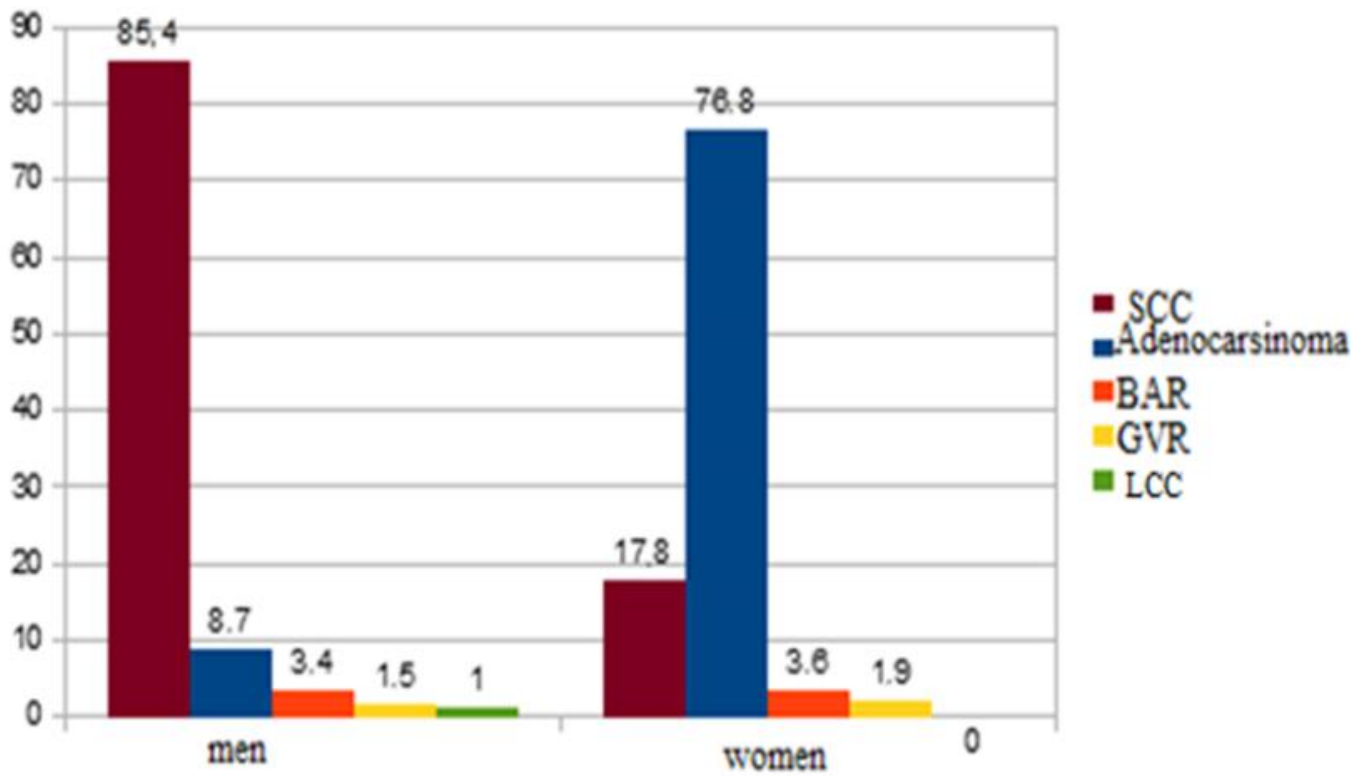

Fig. 3. Distribution of histological types of NSCLC among patients depending on gender: SCC - squamous cell carcinoma; BAR - bronchoalveolar cancer; GVR - glandular squamous cell carcinoma; LCC - large cell carcinoma

histological forms of NSCLC in the patients depending on gender. As the analysis showed, most men had the squamous type of NSCLC, which was diagnosed in $85.4 \%(\mathrm{n}=$ 176) patients, and adenocarcinoma was identified in 18 $(8.7 \%)$ cases. The remaining forms of NSCLC were found in single patients. In female patients, the most common form of NSCLC was pulmonary adenocarcinoma, which was diagnosed in $86(76.8 \%)$ cases, and the rate of squamous cell cancer was $17.8 \%(n=20)($ Fig. 3$)$. The results of the analysis of the occurrence of various morphological types of NSCLC showed their dependence on gender.

\section{Indications for Molecular Genetic Tests in Patients With NSCLC}

According to international and Kazakhstan standards for the diagnosis and treatment of NSCLC, after histological verification of the diagnosis, the next step should include an IHC study to determine the expression of the ALK gene and in its absence and a PCR study to determine the EGFR gene status. This approach was obligatory at the time of this study (9). According to previous results, the number of patients who had indications for IHC studies for the diagnosis of NSCLC; namely, adenocarcinomas, glandular squamous cell carcinomas, and bronchoalveolar cancer, was $120(37.7 \%)$ of 318 patients with NSCLC.

In addition, IHC studies to clarify the histotype of the tumor should be performed in patients with a morphological conclusion of "malignant process" and "undifferentiated cancer." This pool of patients was $11.7 \%$ (45/385). Therefore, the proportion of patients who required IHC studies was 165 (42.9\%), followed by a PCR study. In practice, IHC studies were performed in $50.9 \%$ (84/165) of patients only, while in $6(7.1 \%)$ cases, high expression of the ALK gene was identified. FISH was performed in 3 patients only and confirmed the deletion of the ALK gene.

According to the international algorithm for the diagno- sis of pulmonary adenocarcinoma, the next step in the obligatory study of tumor characteristics is a PCR study. In general, if we focus on the known data on the incidence of mutations in the ALK gene, which does not exceed 5\% in patients with NSCLC, the proportion of patients who require the next step should be about 157 (95.1\%). Valid data on genetic tests were present in 206 cases only. Of these, data on a PCR study are presented only for 35 (17\%) of 206 patients; and the study was not performed on the remaining $83 \%$ of the cases (Table 2).

The rate of the EGFR gene mutation in patients with a verified diagnosis of "lung adenocarcinoma" is known to reach $40 \%$ in the Asian population. The data obtained is consistent with the results of large epidemiological studies. However, it is obvious that the coverage of patients with NSCLC who require molecular genetic testing is low $(17 \%)$ and does not correspond to the modern algorithm for NSCLC diagnosis.

Of the 35 patients diagnosed with "lung adenocarcinoma" who underwent a PCR study, the majority were women, $26(74.3 \%)$ and $7(25.7 \%)$ were men. Mutations in the EGFR gene were detected in $71.4 \%(10 / 14)$ of women and only in $4(27.6 \%)$ men. Therefore, as shown by a retrospective analysis, as of 2015-2017, in the Republic of Kazakhstan, there was no correct understanding of the importance of molecular genetic methods for the diagnosis of NSCLC, allowing individualization of treatment.

Table 2. The results of pcr studies to determine the status of the EGFR gene

\begin{tabular}{lcc}
\hline PCR Findings & Number of Cases & $\%$ \\
\hline No EGFR mutations & 21 & 60 \\
EGFR mutations: & 14 & 40 \\
$\quad$ - Exon 19 del mutation in & 6 & 17.1 \\
EGFR exon 19 & 8 & \\
$\quad$ - Exon 21 L858R mutation & 35 & 22.9 \\
Total & & $100 \%$ \\
\hline
\end{tabular}


This fact is confirmed by the low level of IHC and PCR studies, $50.7 \%$ and $17 \%$, respectively.

The Role of Pharmacotherapy in Patients With NSCLC in Clinical Practice in the Republic of Kazakhstan

One of the main treatments for LC are surgical interventions. In our study, data on the presence or absence of this step in antineoplastic therapy is presented for 397 (93.9\%) patients; there are no data for $26(6.1 \%)$ cases. According to the available information, $141(35.5 \%)$ patients were operated on. In most cases, $256(64.5 \%)$ patients received conservative treatment. The number of patients subjected to surgical intervention correlates with the number of patients with early stages of the disease; stage I to II LC was identified in 158 of 420 (37.4\%) cases. More than half of the patients $(64.5 \%)$ needed pharmacotherapy, which again confirms the importance of molecular genetic testing when choosing antineoplastic therapy.

Data on radiation therapy are presented for 123 (29.1\%) patients, of which this type of treatment was not used in $16(13.0 \%)$ cases. Therefore, it can be assumed that the radiation therapy at different stages was used in 107 $(25.3 \%)$ patients. The lack of data in $300(69.9 \%)$ patients is more likely an evidence of the lack of radiation therapy.

In $35(32.7 \%)$ cases, radiation therapy was done in the main focus in the standard mode of 2 Gy of 5 fractions per week, with a total focal dose (TFD) of $40 \mathrm{~Gy}$. In 44 $(41.1 \%)$ patients, the treatment was done according to a radical program; they received radiation therapy at a total focal dose (TFD) of 50-60 Gy. In 28 (26.2\%) cases, radiation therapy was adjuvant and/or palliative in the form of irradiation of metastatic foci in the bones or the brain. In these cases, the TFD of radiation therapy ranged from 10 to $30 \mathrm{~Gy}$ for bone metastases and lymph nodes, and $70 \mathrm{~Gy}$ for the central nervous system.

We analyzed pharmacotherapy that was used for NSCLC in 318 patients. The total number of courses of pharmacotherapy (chemotherapy, targeted therapy) was 1198. Patient distribution according to the number of received courses of pharmacotherapy is presented in Figure 4.

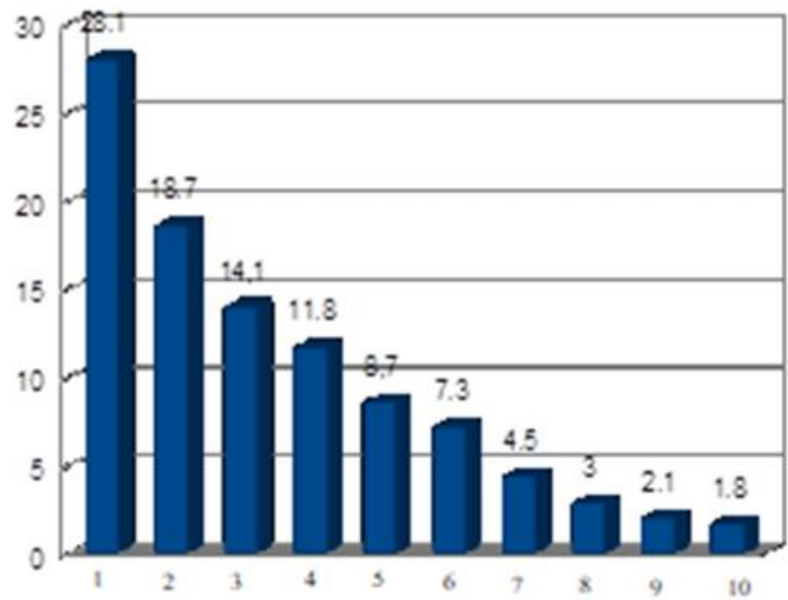

As mentioned above, IHC and FISH studies performed in $84(50.9 \%)$ of 165 patients showed high expression of the ALK protein and dislocation in its gene in $6(7 \%)$ patients. PCR studies to identify EGFR gene mutations were performed in 35 patients only, while in 14 (40\%) patients, mutations were detected in 19 and 21 exons.

Patients with a proven mutation of the above genes received targeted therapy, but not in full. Of 6 patients with high ALK expression, 1 patient received crizotinib. At the time of completion of the study, he had received the drug for 7 months.

Patients with the EGFR gene mutations (14 patients) were prescribed therapy with tyrosine kinase inhibitors: gefitinib and erlotinib. According to the study data, erlotinib therapy included 27 courses and gefitinib 36 courses. Each patient took one of the drugs for an average of 7 months at least.

Most patients with NSCLC received traditional chemotherapy. Basic chemotherapy for NSCLC is based on platinum-based drugs. However, the analysis of treatment for 2015-2018 showed that the proportion of platinum-based drugs in the total cytostatic therapy was $74 \%$. At the same time, cisplatin was preferred and was prescribed by physicians in $716(59.4 \%)$ courses of therapy, while carboplatin was used in $176(14.7 \%)$ cases only, despite its lower toxicity and better tolerability.

A rather low level of taxanes prescriptions in NSCLC should be noted. Paclitaxel was administered in 39 (3.3\%) patients and docetaxel in 107 (8.9\%) courses of treatment. On the other hand, the regimes using anthracyclines and cyclophosphamide were quite unexpected, accounting for $4.4 \%$ (52) and 9.4\% (113) of all treatment regimens, respectively. Administration of gemcitabine was observed in $235(19.6 \%)$ cases and vinorelbine in $40(3.3 \%)$ cases.

Surprisingly, there were no references to the use of pemetrexed in patients with adenocarcinoma in any medical record. However, pemetrexed is the drug of choice with proven high efficacy for the first-line therapy in patients with adenocarcinoma. Moreover, modern treatment standards for patients with pulmonary adenocarcinoma also include maintenance therapy with pemetrexed in pa-

Fig. 4. Distribution of NSCLC patients by the number of received courses of antineoplastic pharmacotherapy 
tients with regression and/or stabilized disease $(10,11)$.

Therefore, one-third of the patients underwent surgical interventions. In this study, we did not assess the type and extent of surgical interventions; however, there is no doubt that all $141(35.5 \%)$ patients underwent radical surgery. Unambiguously, this number of surgical interventions for NSCLC included patients who underwent surgical interventions for diagnostic purposes and in some cases as palliative interventions.

As mentioned above, radical radiation therapy as a consolidating stage was done in one-fourth of the patients with NSCLC (79/318); that is, in $24.8 \%$ of cases only.

Obviously, most of the patients needed antineoplastic drug therapy. However, the analysis results showed that antineoplastic drugs in most patients were chosen without strict adherence to international and republican protocols. Such a conclusion can be made based on a low coverage of patients with NSCLC with genetic studies, and as a result, low coverage with targeted therapy in these patients. Despite the fact that all 14 patients with confirmed EGFR gene mutations received targeted therapy (erlotinib/gefitinib), their proportion in the total number of patients with NSCLC was only 4.4\% (14/318) (12-15).

To obtain an objective assessment of the importance of the right treatment option, we studied the long-term survival results depending on the presence or absence of IHC and genetic tests.

\section{Assessment of overall patient survival}

Of 209 patients included in the study, 124 (59.3\%) were alive at the time of data collection. Median survival was 42.4 months; SE was 4.7 months, with 95\% CI, 33.251.6. Figure 5 shows the survival curve for all monitored patients.

Unfortunately, we cannot give an objective assessment of the causes of mortality; that is, show cancer-specific survival for objective reasons. The main cause of death was not specified in some patients; therefore, we only presented overall survival. Considering the causes of mortality in cancer patients, it can be assumed that during the first year, patients normally die because of the advancement of the disease (stage IV disease) or complications, including complications of the treatment. A 1-year survival was $75 \%$ (SE, 3.3). A 2-year survival rate was $62 \%$ (SE, 4.2) and a 3-year survival rate was 58\% (SE, 4.1).

In our study, immunohistochemical status was not determined in $157(75.1 \%)$ of 209 patients; in 52 (24.9\%) cases, an IHC study was performed with additional data on tumor histotype, ALK and TTF status, which allowed more differential approach in pharmacotherapy of NSCLC.

Among the patients with uncertain immunohistochemical status, $87(55.4 \%)$ patients were alive, with a median survival of 37.9 months (SE, 7.3 months; 95\% CI, 23.552.2). Among the patients with a certain immunohistochemical status, $37(71.2 \%)$ patients were alive, with a median survival of 58.3 months (SE, 0 months, 95\% CI, 58.3). A 1-year survival rate among the patients with uncertain immunohistochemical status was $71 \%$ (SE 4) a 2year survival rate was $57 \%$ (SE 4), and a 3-year survival rate was $53 \%$ (SE, 4). A 1-year survival rate among the patients with certain immunohistochemical status was $82 \%$ (SE, 6), a 2-year survival rate was $75 \%$, (SE, 6), and a 3-year survival rate was $72 \%$ (SE, 7) (Fig. 6).

Therefore, additional data that can be obtained using a IHC study provides additional data on the morphological picture of the malignant process in the lungs. This, in turn, helps to choose an appropriate method of treatment. Therefore, an uncertain immunohistochemical status is a risk factor. A significant difference in median survival is observed and statistical differences between these parameters were significant, $\chi 2=4.0, P=0.046$. It based on $\log$ rank test (Fig. 6).

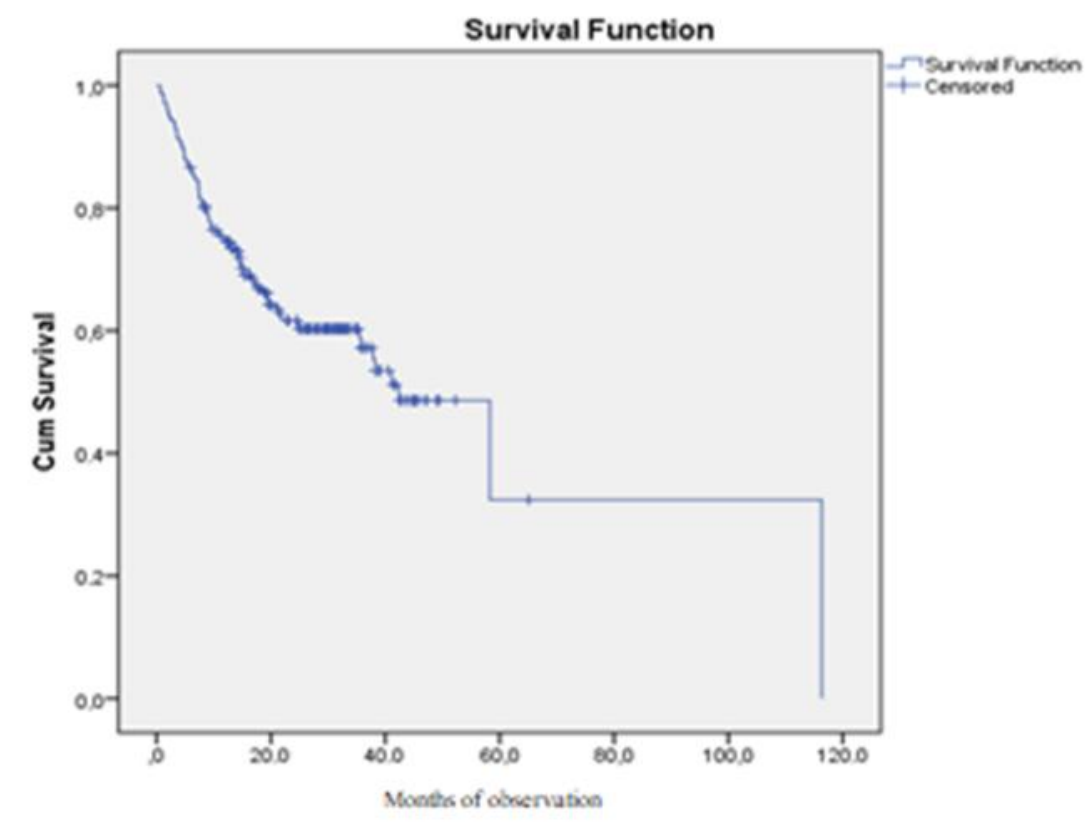

Fig. 5. Survival curve for all patients using the Kaplan-Meier method 


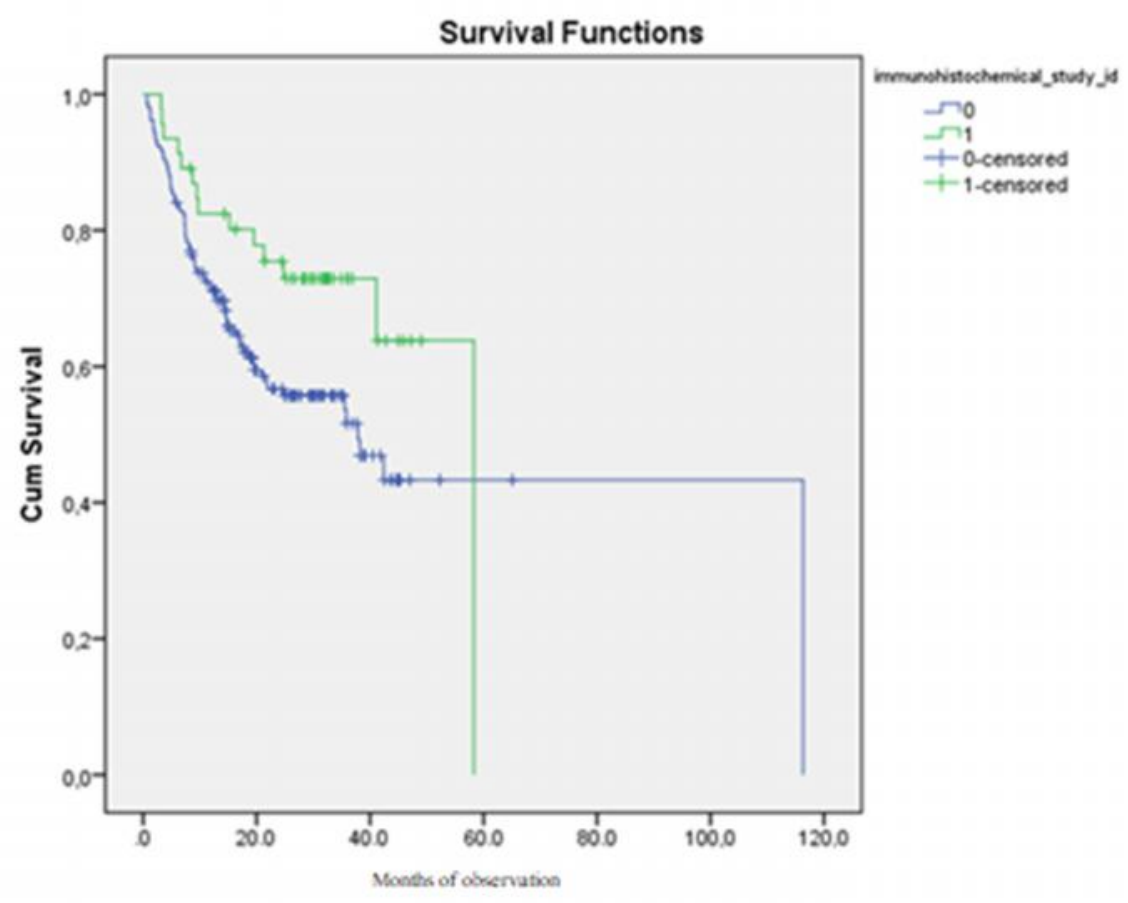

Fig. 6. Survival curve for all patients depending on immunohistochemical status according to the Kaplan-Meier method

\section{Genetic Status as a Prognostic Factor in the Survival of Patients With NSCLC}

In our study, the EGFR gene status was unknown in 195 $(93.8 \%)$ of 209 patients; genetic status was determined in $14(6.2 \%)$ patients only, which allowed the prescription of targeted first-line therapy (erlotinib and/or gefitinib) in these patients. In other cases, with unknown status of the epidermal growth factor gene, patients received chemotherapy. Targeted therapy with tyrosine kinase inhibitors in the absence of data on the EGFR gene status was prescribed only in the second or third line of therapy, when the possibilities of cytostatics were exhausted. In this case, targeted therapy was palliative in nature and could be assessed as "despair" therapy. This affected the efficacy of treatment. The analysis of patient survival based on the available data on the EGFR gene status confirmed the importance of the result of a genetic study for the choice of treatment strategy.

Among the patients with undetermined genetic status who received cytostatic therapy, 115 (59\%) patients were alive. Among the patients with determined genetic status who were prescribed the first-line targeted therapy with epidermal growth factor tyrosine kinase inhibitors, 9 (64.3\%) patients were alive.

A 1-year survival rate among the patients with undetermined genetic status during chemotherapy was $74 \%$ (SE, 3 ), a 2-year survival rate was $61 \%$ (SE, 4), and a 3-year survival rate was $57 \%$ (SE, 4).

Adequate targeted therapy based on the results of molecular genetic tests and compliance with the principles of individual treatment, even in a small number of patients, resulted in an increase in 1-year survival the rates up to $86 \%$ (SE, 9); a 2-year survival rate up to $71 \%$ (SE, 12), and a 3 -year survival rate up to $71 \%$ (SE, 12).
Therefore, we believe that noncompliance with the recommendations on the sequence of morphological and molecular genetic stages of the diagnosis of NSCLC and failure to follow the principles of an individual approach in the selection of pharmacotherapy for patients with NSCLC are risk factors. A significant difference in survival was observed, despite the fact that statistical difference between these parameters was not significant, $\chi 2=0.87 ; \mathrm{P}=0.035$. This fact is probably associated with a small number of observations (Fig. 7).

One of the key prognostic factors affecting survival is the stage of the neoplastic process. There was a significant difference in median survival; statistical difference between these parameters was significant, $\chi 2=51.1 ; \mathrm{P}=0.001$.

In our study, 70 (33.5\%) patients of 209 did not have surgical treatment, $118(56.5 \%)$ underwent surgical treatment, and $21(10.0 \%)$ did not have reliable data. Among the patients without surgical treatment, 29 (41.4\%) patients were alive, with a median survival of 17.7 months (SE, 2.4 months; 95\% CI, 12.9- 22.5). Among the patients who underwent surgical treatment, $85(72.0 \%)$ patients were alive, while the median survival was 58.3 months (SE, 21.7 months; 95\% CI, 15.8-100.8). Among patients with no reliable data on surgical treatment, $10(47.6 \%)$ patients were alive with a median survival of 23.2 months (SE, 3.5 months; 95\% CI, 16.3- 30.1) (Fig. 8).

A surgical stage in the treatment of patients with NSCLC resulted in the best survival rates, and the absence of surgical treatment was a risk factor for the patient. There was a significant difference in median survival, which was statistically significant, $\chi 2=29.1 ; P=0.001$. The lack of radiological treatment was a risk factor for the patient. There was a significant difference in median survival: overall survival was worse in the absence of radio- 


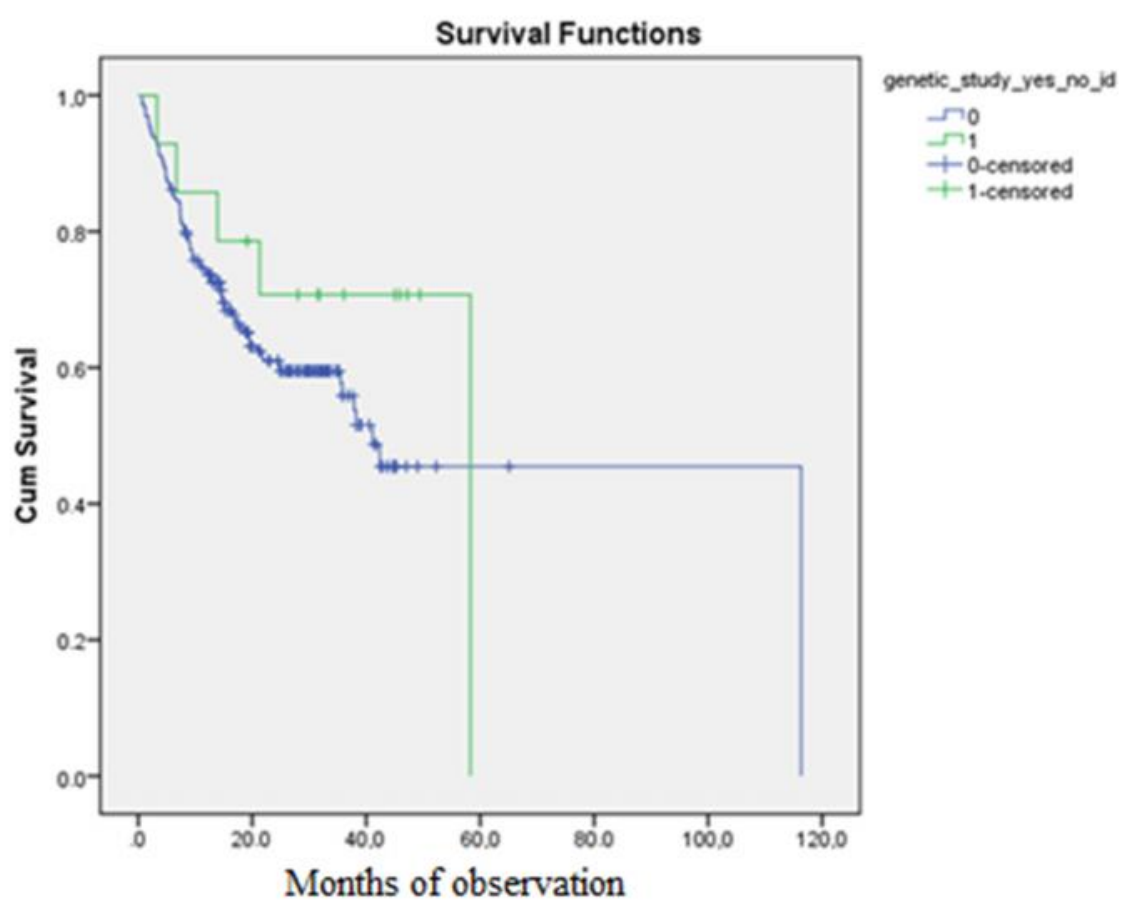

Fig. 7. Survival curve for all patients depending on genetic status using the Kaplan-Meier method

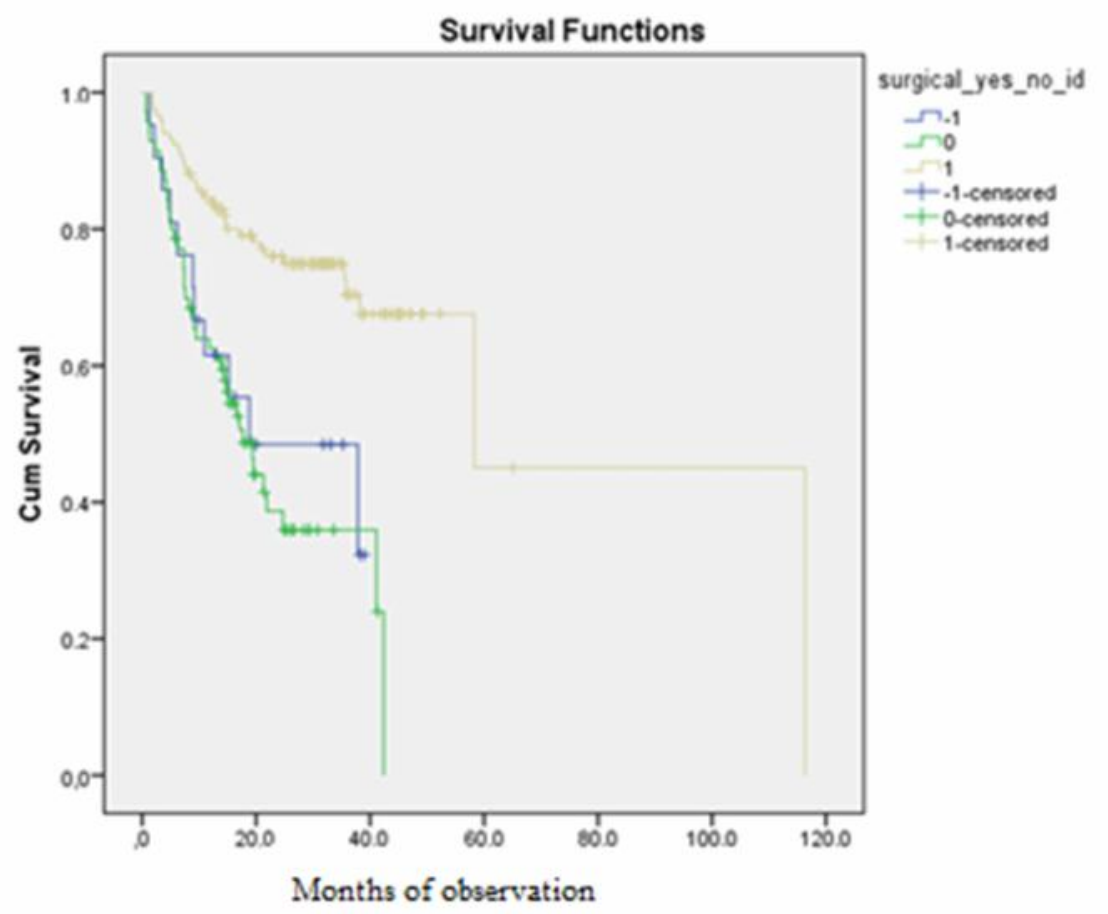

Fig. 8. Survival curve for all patients depending on surgical treatment using the Kaplan-Meyer method

logical treatment. However, the difference between these parameters were statistically insignificant, $\chi 2=0.86$; $\mathrm{P}=0.036$ (Fig. 8).

Gender was not a risk factor. There was a significant difference in median survival, but statistically the difference between these parameters was insignificant, $\chi 2=0.99$; $\mathrm{P}=0.032$. Social status did not affect the survival rate of patients with NSCLC. There was no significant difference

in median survival, which was confirmed statistically, $\chi 2=0.8 ; \mathrm{P}=0.032$.

A low baseline patient status was a risk factor of low treatment efficacy and poor prognosis for NSCLC patients. There was a significant difference in median survival; and the statistical difference between these parameters was significant, $\chi 2=31.6 ; \mathrm{P}=0.001$ (Fig. 9) (16). 


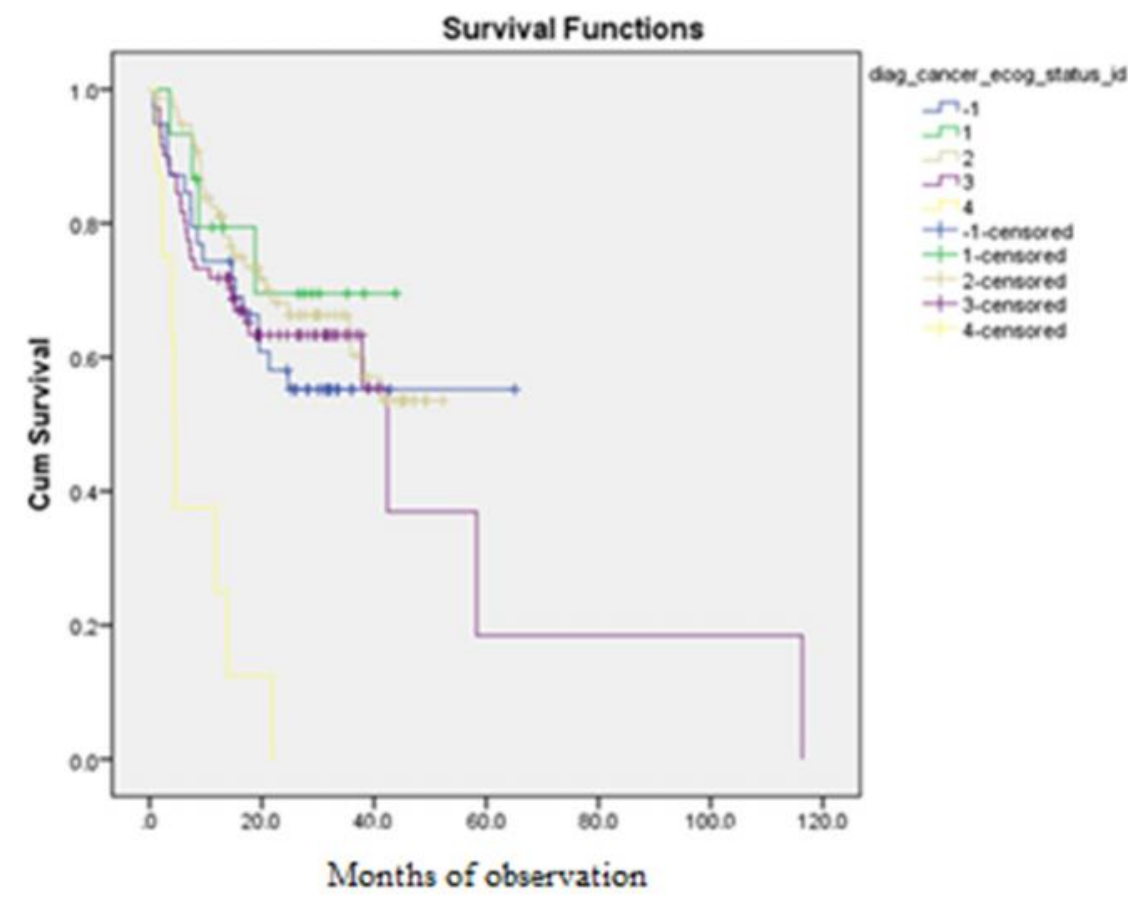

Fig. 9. Survival curve for all patients depending on eastern cooperative oncology group (ECOG) scale status using the Kaplan-Meier method.

\section{Discussion}

According to the latest recommendations of international oncological associations, as well as the Kazakhstan Republican Protocols for the Diagnosis and Treatment of Lung Cancer, the range of molecular genetic markers in the diagnosis of NSCLC has expanded, as has the range of patients who require such studies $(17,18)$.

Considering that the modern approach to pharmacotherapy of NSCLC is based both on the results of morphological studies and determination of the histotype of the tumor, as well as molecular genetic data, to determine the status of certain genes, the role of chemotherapy is inferior to targeted therapy in proven mutation or deletion of the EGFR and ALK genes $(19,20)$.

According to published data, the proportion of patients with EGFR mutation status in the Asian population is $30 \%$ to $40 \%$ of cases (12-15). The data on the rates of radiation therapy once again emphasizes that most of the patients included in the retrospective analysis required pharmacotherapy, the choice of which should be determined based on the histological type of the tumor and on the EGFR and ALK gene status, which was available in Kazakhstan at the time of this study (21-23).

\section{Conclusion}

Based on a retrospective analysis of the diagnosis and treatment approaches in the treatment of LC in Kazakhstan, the following conclusions can be made: a comparative analysis of the compliance of oncologists from various regions of the republic with molecular genetic testing as an essential component of the diagnosis of NSCLC showed that the coverage of patients with IHC and PCR studies in this country is low. At the same time, the majority of studies were performed on patients in Almaty and only $35.8 \%$ of IHC studies were performed in other 5 regions.

Moreover, the majority of patients received drug therapy, the choice of which was determined mainly by the results of morphological data without assessing the genetic characteristics of the tumor; that is, without following the principle of a personalized approach.

The risk factors for patients with NSCLC were the lack of IHC status data and the choice of therapy based on the results of genetic status, which showed a significant but unreliable difference due to the small number of observations. Favorable prognostic factors include the surgical component, the presence of which significantly improves the survival rates of patients with NSCLC.

\section{Acknowledgement}

The authors would like to acknowledge the contribution of the pharmaceutical company AO "Nobel AFF" for the allocated research grant under agreement No. 2 dated April 28, 2018 on rendering services for the implementation of the project "Retrospective observational clinical study of the efficacy and safety of tyrosine kinase inhibitors (Erlotinib, Gefitinib) in the treatment of metastatic NSCLC in routine clinical practice in the Republic of Kazakhstan".

\section{Conflict of Interests}

The authors declare that they have no competing interests.

\section{References}

1. Calkins H, Reynolds MR, Spector P. Treatment of atrial fibrillation with antiarrhythmic drugs or radiofrequency ablation: Two systematic literature Kaidarova DR, Auezova ET, Chingisova ZhK, Seisenbaeva GT, Azhmagambetova AE, Zhylkaidarova AZh. Indicators of the 
oncological service of the Republic of Kazakhstan for 2015. Almaty: Nauka, 2016

2.Pavlakis N. As cancer therapy becomes more complex, we must enhance our professional standards. J Pharm Prac Res. 2020;50(6):461-465.

3.D'Addario G, Früh M, Reck M, Baumann P, Klepetko W, Felip E. Metastatic non-small-cell lung cancer: ESMO clinical practice guidelines for diagnosis, treatment and follow-up. Ann Oncol. 2010;21(5):v116-v119.

4. Travis WD, Brambilla E, Burke AP, Marx A, Nicholson AG. Introduction to the 2015 world health organization classification of tumors of the lung, pleura, thymus, and heart. $\mathrm{J}$ Thor Oncol. 2015;10(9):1240-1242.

5. Sebastian M, Rydén A, Walding A, Papadimitrakopoulou V. Patientreported symptoms possibly related to treatment with osimertinib or chemotherapy for advanced non-small cell lung cancer. Lung Cancer. 2018;122:100-106

6. Clinical protocol for the diagnosis and treatment of lung cancer Recommended by the Expert Council of the Republican State Enterprise on the REM "Republican Center for Health Development" of the Ministry of Health and Social Development of the Republic of Kazakhstan dated October 30, 2015. Protocol No. 14 [cited 2020 Oct 19]. Available from: https://onco.kz/wp-content/uploads/2017/12/22. pdf

7. Smagulova GK, Tleukhanov ST. Development of an algorithm for molecular genetic diagnostics in non-small cell cancer in the Republic of Kazakhstan. Almaty: KazNU named after Al-Farabi, 2017.

8. Declaration of Helsinki by the World Medical Association. 59th General Assembly of the WMA, Seoul, Republic of Korea. 2008 [cited 2020 Oct 19]. Available from: http://rostgmu.ru/wpcontent/uploads/2014/12/WMA_Helsinki.pdf

9. Gaur P, Bhattacharya S, Kant S, Kushwaha R, Singh G, Pandey S. Association of cytokines levels with epidermal growth factor receptor mutation in lung cancer patients. J Canc Res Ther. 2020;16(4):811815.

10. Paz-Ares L, de Marinis F, Dediu M, Thomas M, Pujol JL, Bidoli P, et al. Maintenance therapy with pemetrexed plus best supportive care versus placebo plus best supportive care after induction therapy with pemetrexed plus cisplatin for advanced non-squamous non-small-cell lung cancer (PARAMOUNT): a double-blind, phase 3, randomised controlled trial. Lancet Oncol. 2012;13(3):247-55.

11. Halvorsen TO, Stokke K, Killingberg KT, Raj SX, Sørhaug S, Brustugun OT, et al. Randomized phase III trial comparing switchmaintenance pemetrexed with observation followed by pemetrexed at progression in advanced NSCLC. Acta Oncol. 2020;59(9):1051-1057.

12. Suda K, Mitsudomi T. Development of personalized treatments in lung cancer: Focusing on the EGFR mutations and beyond. Lung Canc: Targ Ther. 2013;4:43-53.

13. Tezel GG, Şener E, Aydın Ç, Önder S. Prevalence of epidermal growth factor receptor mutations in patients with non-small cell lung cancer in Turkish population. Balkan Med J. 2017;34(6):567-571.

14. Tjulandin S, Imyanitov E, Moiseyenko V, Ponomarenko D, Gurina L, Koroleva I, et al. Prospective cohort study of clinical characteristics and management patterns for patients with non-small-cell lung cancer in the Russian Federation: EPICLIN-Lung. Curr Med Res Opin. 2015;31(6):1117-27.

15. Zhao FN, Zhao YQ, Han LZ, Xie YS, Liu Y, Ye ZX Clinicoradiological features associated with epidermal growth factor receptor exon 19 and 21 mutation in lung adenocarcinoma. Clin Radiol. 2019;74(1);80.e7-80.e17.

16. West H, Jin JO. Performance status in patients with cancer. JAMA Oncol. 2015;1(7):998

17. Koneva ES, Omelchuk NN, Kuzmenko L, Kosova I, Afanasyeva NV. Introduction of electronic cancer patient registries. Prensa Med Argent. 2019;105(9):546-555.

18. Romashin OV, Liadov KV, Makarova MR, Koneva ES, Preobrazhenski VI, Chudimov VF. The development of physical education as a basic instrument of rehabilitative treatment, remedial medicine, and goal-oriented health promotion for the benefit of man. Vopros Kurort Fizioter Lech Fiz Kult. 2013;1:39-43.

19. Lipatov VA, Lazarenko SV, Sotnikov KA, Severinov DA, Ershov MP. To the issue of methodology of comparative study of the degree of hemostatic activity of topical hemostatic agents. Nov Khir 2018;26(1):81-95.

20. Omertayeva D, Muravlyova L, Ponomaryova O, Molotov-
Luchanskyi V, Bakirova R, Klyuev D, et al. The level assessment of extracellular nucleic acids in the blood of pregnant women with chronic hypertension with superimposed preeclampsia. Open Access Maced J Med Sci. 2020;8(B):514-8.

21. Madzhuga AG, Kislyakov PA, Abdullina LB, Serdakova KG, Sadovnikova TI. Human health as a multidimensional phenomenon: Approaches to study and phenomenology. J Pharm Sci Res. 2018;10(11):2972-5.

22. Burkitbaev JK, Ramileva IR, Turganbekovaa A, Baimukasheva DK, Imashpaev D, Isaev TK. The character of distribution of HLA specificities in oncological patients. Klin Labor Diag. 2017;62(5):2825.

23. Maslak K, Favara-Scacco C, Barchitta M, Agodi A, Astuto M, Scalisi R, et al. General anesthesia, conscious sedation, or nothing: Decision-making by children during painful procedures. Pediatr Blood Canc. 2019;66(5):e27600. 